
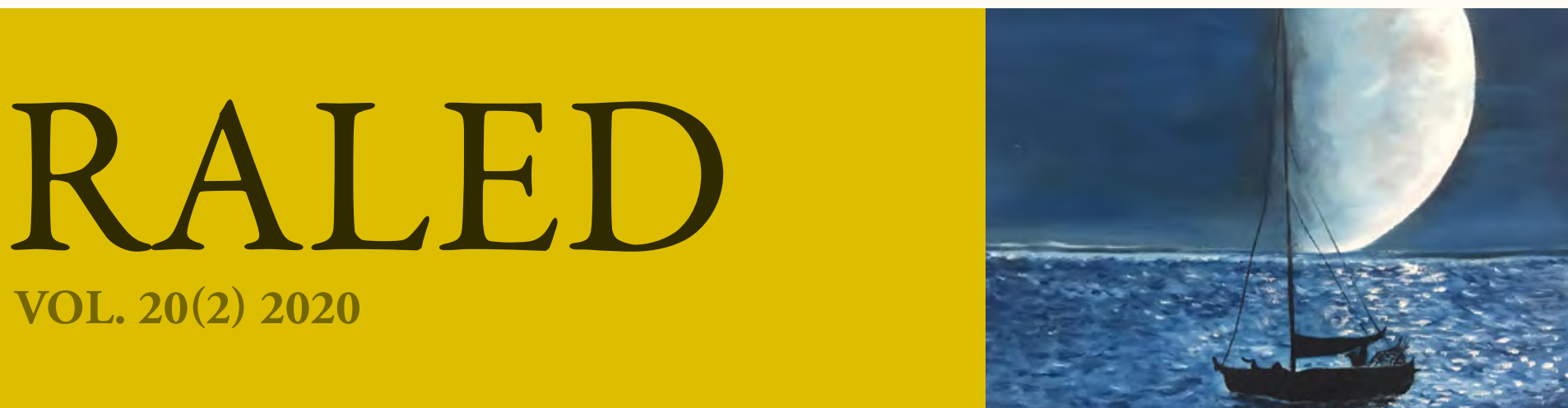

\title{
Exploración del cuantificador "todo" como recurso de GRADACIÓN de valoraciones en un corpus de espańol oral conformado por un grupo de madres de Santiago de Chile ${ }^{1}$
}

Analysis of the quantifier "todo" as a resource of GRADUATION of appraisals in a spoken Spanish corpus conformed by a group of mothers from Santiago, Chile

\section{CONSUELO GAJARDO}

Pontificia Universidad Católica de Chile Chile

\section{CLAUDIA CASTRO}

Pontificia Universidad Católica de Chile Chile

Recibido: 31 de julio de 2020 | Aceptado: 28 de septiembre de 2020 DOI: $10.35956 /$ v.20.n2.2020.p.69-91 
Este artículo describe el uso del cuantificador todo como recurso de GRADAción (Martin y White 2005; Hood 2010, 2019) en un corpus de español oral compuesto por los relatos de un grupo de madres de Santiago de Chile. Los resultados muestran que el cuantificador todo realiza significados congruentes con su función cuantificadora y, adicionalmente, despliega realizaciones menos congruentes, donde cumple una función intensificadora, como también la de graduar una categoría semántica en términos de prototicipidad y de completitud. El análisis demuestra que estas realizaciones de todo como recursos de GRADACión tienen un rol significativo en la evocación de valoraciones involucradas en la construcción de identidades maternas particulares.

PALABRAS CLAVE: Gradación. Sistema de Valoración. Cuantificador todo. Identidad Materna.

\section{RESUMO}

Este artigo examina o emprego do quantificador 'todo' como recurso de GRADAÇÃo (Martin e White 2005; Hood 2010, 2019) em um corpus oral, constituído por entrevistas em profundidade com um grupo de mães de Santiago do Chile. Os resultados mostram que o quantificador 'todo' realiza significados que são congruentes com a sua função quantificadora e, adicionalmente, exibe realizações menos congruentes, tais como uma função intensificadora, mas também a função de graduar uma categoria semântica em termos de prototipicidade e integralidade. A análise mostra que essas realizaçóes do quantificador 'todo' como recurso de gradação têm um papel importante em valoraçôes envolvidas na construção de identidades maternas particulares.

PALAVRAS CHAVE: Gradação. Sistema da Avaliatividade. Quantificador 'todo'. Identidade materna.

\section{ABSTRACT}

This article describes the use of the quantifier todo as a GRADUATION resource (Martin and White 2005; Hood 2010, 2019) in a spoken Spanish corpus, composed of the life stories of a group of mothers from Santiago, Chile. The results show that the quantifier todo realizes meanings that are congruent with its quantifying function, and additionally, it exhibits less congruent realizations, such as an intensifying function, and also that of graduating a semantic category in terms of prototipicity and completion. The analysis demonstrates that these realizations of the quantifier todo as a GRADUATION resource have a significant role in the invocation of appraisals involved in the construction of particular mother identities.

KEYWORDS: Graduation. System of Appraisal. Quantifier todo. Mother identity.

1 Este artículo se ha desarrollado en el marco del Proyecto Fondecyt Regular 1170331, "El lenguaje de la valoración en español: descripción y sistematización de recursos para construir intersubjetividad”. 


\section{Introducción}

El estudio de la evaluación en el discurso, como la expresión de actitudes y la negociación de posturas ideológicas, ha tenido un gran desarrollo entre analistas del discurso en las últimas décadas (Bolívar 1994; Hunston y Thompson 2000; Alba Juez y Thompson 2014). Un enfoque interpersonal útil para el análisis de la evaluación corresponde al sistema de valoración (Martin 2000; Martin y White 2005; Hood y Martin 2005; Hood 2010, 2019), basado en la Lingüística Sistémico Funcional, que constituye una aproximación discursiva semántica de los recursos para evaluar la experiencia social, ofreciendo una herramienta lingüística detallada para analizar la intersubjetividad, la identidad y los posicionamientos en el discurso (Martin y White 2005).

Las herramientas del sistema de valoración han resultado productivas para los analistas del discurso, especialmente para quienes trabajan desde una perspectiva crítica, en tanto permite relevar las posturas ideológicas que se (des) legitiman en los diversos intercambios lingüísticos en la vida social, colaborando con un mayor entendimiento de los fenómenos sociales (Oteíza y Pinuer 2019). Este sistema ha sido ampliamente aplicado en Latinoamérica, entre analistas críticos del discurso que trabajan en el campo de la memoria en períodos de dictadura (Achugar 2008, 2016; Oteíza 2009, 2011; Giudice 2010; Oteíza y Pinuer 2012, 2016) y también en la representación de la violación de los derechos humanos y los conflictos armados en la prensa (Bolívar 2007; Kaplan 2012; Pascual 2014, 2017).

Al interior del sistema de VALORACIÓN, el subsistema de GRADACIÓn es considerado por Martin y White (2005) como central para el modelo, debido a su función fundamental en la intensificación o la atenuación de las actitudes y en los mayores o menores grados de involucramiento del hablante. Dentro de los desarrollos latinoamericanos en este subsistema destacan las contribuciones para el portugués brasileño en relación con la intensificación de las actitudes (Vian Jr. 2008) y para el español en relación con la construcción simbólica del tiempo en el discurso histórico (Oteíza y Pinuer 2013).

A pesar de estas valiosas contribuciones, la tipologización, desde la perspectiva de la Lingüística Sistémico Funcional, de los recursos interpersonales para realizar GRADACión en español es todavía escasa, por lo que se requiere una descripción más completa de los recursos léxico-gramaticales en español para intensificar o atenuar significados ideacionales y valorativos, así como también de la manera en que la GRADACión opera en esta lengua en relación con la evocación de las actitudes. Por lo tanto, en este artículo nos proponemos describir el uso del cuantificador todo como recurso para construir GRADACIÓN en el discurso de un grupo de madres de los estratos socioeconómicos alto, medio y bajo de Santiago de Chile ${ }^{2}$. Nos centramos en el cuantificador todo debido a su alta ocurrencia en el español oral (Arce Castillo 1999), aspecto vinculado con su característica sintáctica de poseer múltiples posibilidades combinatorias y posicionales (Suárez Fernández 2012).

Como analistas del discurso que exploran la manera en que las prosodias valorativas colaboran con la legitimación de distintas identidades e ideologías, en este caso particular, de la maternidad,

2 En este trabajo utilizamos un corpus de espańol oral compuesto por los relatos de vida de madres de tres grupos socioeconómicos de Santiago de Chile. Sin embargo, en este artículo no abordaremos la construcción de la identidad materna según grupo socioeconómico. 
y cómo ellas reproducen desigualdades de género (Gajardo 2017; Gajardo y Oteíza 2017; Gajardo

2018; Gajardo en prensa), nos planteamos describir los patrones de GRADACIón que colaboran con la construcción de posicionamientos y actitudes más o menos intensificadas o cuantificadas en un corpus de español oral en el campo de la identidad materna.

Mediante los objetivos propuestos en este trabajo, buscamos contribuir en un área de todavía escasa exploración, y aportar a la descripción y sistematización de los recursos léxico-gramaticales y discursivo semánticos que permiten graduar valoraciones en un corpus de español oral.

\section{Fundamentos teóricos}

\subsection{Sistema de VALORACIÓN}

El sistema de valORACión, fundamentado en la arquitectura teórica de la LSF, constituye una orientación discursiva semántica de los recursos lingüísticos para valorar la experiencia social, y proporciona una herramienta lingüística comprehensiva para analizar la intersubjetividad y los posicionamientos ideológicos en el discurso, dando cuenta de los efectos retóricos relacionados con diversas selecciones léxico-gramaticales (Martin 2000; Martin y White 2005). Con base en la estratificación del plano del contenido en la semántica discursiva y en la léxico-gramática (Martin 1992), la evaluación se concibe como funcionando de manera prosódica por sobre los límites de la gramática (Martin 2014, 2019). Así, el sistema de valoración, ubicado en el estrato discursivo-semántico, constituye un sistema más abstracto que el nivel léxico-gramatical, por lo que la valoración se realiza en una amplia variedad de sistemas y recursos léxico-gramaticales, que se acumulan en prosodias valorativas.

El modelo ofrece una sistematización de los recursos para la evaluación y el posicionamiento intersubjetivo en tres subsistemas: COMPROMISO, ACTITUD y GRADACIÓN.

El sistema de COMPROMIso organiza las locuciones para distintos posicionamientos de la voz autorial en un discurso monoglósico (voz autorial única) o heteroglósico (inclusión de posturas y voces alternativas).

El sistema de ACTITUD reúne los recursos semánticos para expresar emociones (subsistema de Afecto), valorar el comportamiento de las personas (subsistema de Juicio) y evaluar los objetos, entidades, situaciones y eventos (subsistema de Apreciación). Estas actitudes pueden ser positivas o negativas y pueden expresarse de manera inscrita (explícitamente) o evocada (implícitamente) en el discurso. Asimismo, las actitudes pueden amplificarse o disminuirse en la Fuerza y agudizarse o suavizarse en Foco, opciones que se organizan en el sistema de GRADACIÓN.

\subsubsection{Gradación y contribuciones al modelo en Latinoamérica}

La GRADACIÓn corresponde al sistema semántico que reúne los recursos que el lenguaje ofrece para amplificar o disminuir las expresiones de actitud, así como también para mostrar distintos niveles de (des) alineamiento con las posiciones intersubjetivas promovidas en un texto (Martin y White 2005). Los significados valorativos se pueden amplificar o mitigar con respecto a la Fuerza, con recursos que gradúan las actitudes en términos de Intensificación o Cuantificación, o 
también se pueden agudizar o suavizar en términos de Foco, por medio de recursos que permiten graduar los límites categoriales de las personas o cosas. La GRADACIÓN en términos de Intensificación puede operar sobre cualidades, procesos o propuestas, mientras que la Cuantificación opera sobre entidades, graduando en términos de número, tamaño, distribución o proximidad. La GRADACIÓN de Foco, o según prototipicidad, aplica comúnmente a categorías que experiencialmente no son graduables, pero colaboran con la evocación de valoraciones (Martin y White 2005; Hood 2010, 2019).

La red sistémica de los recursos para graduar las actitudes en inglés elaborada inicialmente por Martin \& White (2005), fue posteriormente expandida por Hood $(2010,2019)$ en su trabajo sobre los artículos de investigación. En cuanto al subsistema de Fuerza por Cuantificación, la autora señala que no solo se pueden cuantificar entidades, sino que también procesos en cuanto a su Extensión y Frecuencia. Por otro lado, Hood $(2010,2019)$ extiende el subsistema de Foco en las opciones semánticas de Autenticidad y Especificidad, y también elabora los recursos para graduar los procesos en cuanto a niveles de Logro, según Completitud o Actualización. Estos recursos de GRADACIÓN tienen un rol clave en la evocación de valoraciones que se acumulan en el discurso, es decir, permiten seńalar una lectura actitudinal en vez de expresar la actitud explícitamente. La red completa del sistema de GRADACIÓN original (Martin y White 2005) con las aportaciones de Hood $(2010,2019)$ se incluye en la figura 1.

\section{FIGURA 1}

Sistema de Gradación (Hood 2019: 391).

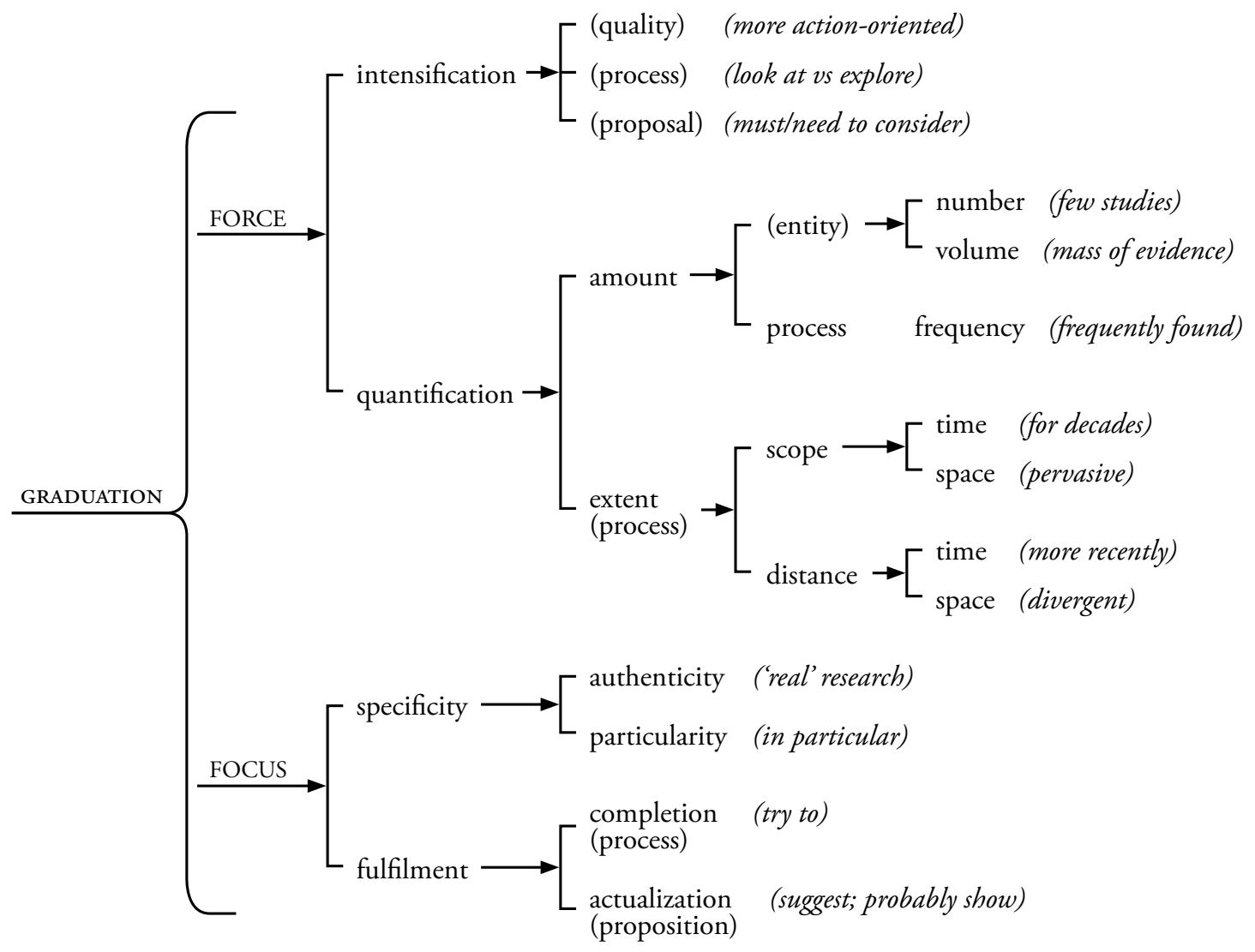


Nuestra descripción de los recursos de GRADACión en el corpus oral analizado responde a los principios de descripción tipológica tal como se plantean desde el modelo de la Lingüística Sistémico Funcional.

Una descripción tipológica informada por la LSF considera, en primer lugar, una clara distinción entre teoría lingüística, como una teoría general sobre el lenguaje, y descripciones de lenguajes particulares (Caffarel, Martin y Matthiessen 2004). Con base en una perspectiva discursiva, la descripción de lenguajes particulares sitúa al texto como punto de partida. Esto implica no solo considerar los aspectos relacionados con el contexto de situación, sino también con el contexto cultural más amplio que concierne a las comunidades de habla involucradas. Así también, se pone énfasis en la selección de textos reales, orales o escritos.

Finalmente, una descripción de este tipo se basa en el significado. El interés está en analizar cómo distintos lenguajes particulares organizan los tres significados principales que se construyen en el lenguaje —ideacionales, interpersonales y textuales_, y cómo estos significados son realizados en los distintos estratos del sistema lingüístico (Kumar 2019).

La tipologización del sistema de GRADACIÓN ha tenido algunos desarrollos en Latinoamérica, específicamente para el portugués brasileño y el español. Vian Jr. (2008) identifica algunos recursos particulares del portugués brasileño, tal como el orden de los elementos del grupo nominal, por medio del cual se puede incrementar o reducir la fuerza de lo expresado, por ejemplo, situando al Epíteto antes del sustantivo al que modifica ("bom agenciador"3 (Vian Jr. 2008: 814)). Lo mismo ocurre con la adición de un prefijo griego o latino a un ítem léxico (“tem un shopping superlegal”) ${ }^{4}$ o con el uso de algunos sufijos ("carrão") para el incremento de la fuerza.

Oteíza y Pinuer (2013), por otra parte, amplían la dimensión temporal en la red sistémica de GRADACIÓn propuesta por Hood (2010), para dar cuenta de la construcción simbólica del tiempo en el discurso histórico en español, aspecto central para legitimar memorias históricas particulares del pasado reciente. Los autores plantean que el tiempo se puede realizar menos congruentemente como un recurso de Intensificación, en términos de aceleración (ej. “desbocamiento"), expectación (ej. “presagiar”) y profundización (ej. “agudizar”), significados realizados principalmente por nominalizaciones y procesos que, a menudo, ensamblan un significado actitudinal.

La descripción tipológica de los recursos de GRADACión en este trabajo se sirve de la descripción de recursos en el rango de la cláusula, el grupo y la palabra para la exploración del papel que juega el cuantificador todo en la gradación de significados actitudinales y experienciales, así como en la constitución de prosodias valorativas en relación con la constitución de identidades maternas ${ }^{6}$ en mujeres chilenas de distintos grupos socioeconómicos.

3 En español, "buen agente".

4 En español, "hay un centro comercial súper bueno".

5 En espańol, "carrazo".

6 Por razones de espacio no podemos ahondar en el enfoque teórico adoptado para el estudio de la identidad materna en esta sección. En términos generales, abordamos la construcción identitaria desde un enfoque 


\subsection{Descripción del cuantificador "todo"}

El cuantificador todo pertenece a los tradicionalmente denominados "cuantitativos" (Alcina Franch y Blecua 1979) o más actualmente llamados "cuantificadores", los que hacen referencia a la cantidad o número que se le atribuye a los objetos designados por el sustantivo a los que se anexan (Alarcos 2000). Con respecto a su naturaleza semántica, todo se considera un cuantificador fuerte, o también cuantificador universal y/o definido (NGLE 2009), que tiene la propiedad de incluir la totalidad de los elementos que integran el conjunto, aunque se presente en singular.

Sintácticamente, todo puede anteceder a un sustantivo, a su vez antepuesto por un determinante ("todo el día") o también puede usarse precediendo a un pronombre personal ("todas nosotras") y a nombres propios que denotan extensión ("todo Chile"), interpretándose como adjetivo. Cuando todo no acompaña a un sustantivo, se le considera pronombre ("todo fluía") (Moliner 2013). En cuanto a sus características morfológicas, el cuantificador todo varía de acuerdo a género y a número, de modo que despliega las opciones de todoltodaltodos/todas, a las que se agrega el neutro todo ("todo le gusta") (NGLE: 2.248).

Se han descrito algunos usos especiales del cuantificador todo, tales como su función genérica y ponderativa. Cuando precede a un sustantivo singular sin determinante puede expresar generalización ("toda infracción será penalizada"). Dentro de sus usos ponderativos, todo puede estar antepuesto a un sustantivo singular precedido del artículo indefinido un/ una para significar que aquello designado por el sustantivo se presenta "en toda su plenitud" (Alcina Franch y Blecua 1979: 644), como en el ejemplo "se ha convertido en toda una mujer". En estos casos todo adquiere un valor análogo a los adjetivos auténtico o verdadero (NGLE 2009). Finalmente, cuando todo acompaña a un adjetivo de cualidad, el cuantificador también adquiere un significado valorativo, siendo análogo a completamente o sumamente, como en el ejemplo "estaba toda feliz" (Moliner 2013: 113).

\section{Marco metodológico}

Este estudio cualitativo se enmarca en un proyecto de investigación más amplio, que analiza la construcción discursiva de la identidad de madre trabajadora en los grupos socioeconómicos alto, medio y bajo de Santiago de Chile, desde una perspectiva de los Estudios Críticos del Discurso (ECD) 7 .

posmoderno que la concibe como una subjetividad dilemática, fragmentada e incompleta, que se construye necesariamente en el discurso (e.g. Giddens 1991; Chouliaraki and Fairclough 1999; Benwell and Stokoe 2006). La identidad materna es particularmente problemática ya que involucra una fractura en la identidad de las mujeres (Laney et al. 2015), además las madres deben conciliar sus aspiraciones individuales y profesionales con las prácticas maternas (LaChance Adams 2014; Gonzálvez 2016) y, finalmente, las madres también deben enfrentar elementos más bien simbólicos como las expectativas sobre una buena madre y los discursos y representaciones sobre la maternidad (Gómez, Arellano y Valenzuela 2017).

7 El grupo socioeconómico de pertenencia resulta un factor clave en la construcción identitaria materna. Las madres del GSE bajo tienden a naturalizar una representación tradicional de la maternidad (Mu- 
En virtud de los objetivos del presente trabajo, esto es, la descripción del uso del cuantificador todo como recurso de GRADACIÓn en el discurso de este grupo de madres, en esta sección se describe la conformación del corpus y el procedimiento de análisis de los datos, sin profundizar en los principios teórico-metodológicos de los ECD ni de los estudios de la identidad, en tanto aproximaciones en la que se sitúa el proyecto mayor. Asimismo, debemos seńalar que, si bien el corpus está estratificado por nivel socioeconómico, en este trabajo esta variable social no fue considerada.

\subsection{Conformación del corpus}

El corpus se conformó por medio de los relatos de vida (e.g. Bertaux 1989, 2005; Ferraroti 1988, 2007) de 12 madres trabajadoras pertenecientes a los grupos socioeconómicos (GSE) alto, medio y bajo de Santiago de Chile ( 4 madres por cada GSE), los que se obtuvieron en 12 entrevistas semiestructuradas, que fueron grabadas y luego transcritas. El detalle socioeconómico de las participantes, así como la manera en que se realizó la estratificación social ${ }^{8}$ y etaria ${ }^{9}$ se encuentra en Gajardo (2017).

\subsection{Análisis de los datos}

En primer lugar, se identificaron todas las ocurrencias del cuantificador todo en el corpus, posterior a lo cual se diseñó una matriz de análisis siguiendo un enfoque trinocular del lenguaje (Halliday \& Matthiessen 2014), por lo que el recurso analizado (cuantificador todo) se describe en cuanto a su función discursiva (sistema de GRADACIÓN) y en cuanto a su realización léxico-gramatical en el rango de la cláusula (análisis de Transitividad), en el rango de grupo y en el rango de la palabra. En el caso del sistema de GRADACIÓn, se utilizaron las categorías semánticas desarrolladas por Hood $(2010,2019)$. La matriz de análisis se presenta a continuación en la tabla 1:

ñoz et al. 2013), mientras que las mujeres del GSE medio cuestionan estos modelos hegemónicos y las representaciones genéricas desiguales (Yopo Díaz 2016). Por último, las madres del GSE alto tienen la posibilidad de autorrealizarse profesionalmente y ejercer mayor autonomía, al delegar el cuidado de niños/as en el servicio doméstico pagado (Undurraga 2013). Los resultados de la construcción identitaria de madre en el GSE bajo se discute en Gajardo y Oteíza (2017). La identidad de madre del GSE medio se reporta en (Gajardo 2018), mientras que la identidad de madres del GSE alto se presenta en Gajardo (en prensa).

8 A grandes rasgos, la estratificación social se llevó a cabo de acuerdo con los niveles sociales adaptados de ICCOM (2007), Rasse, Salcedo y Pardo (2009) y AIM (2015), con los que se eligieron a 4 madres prototípicas de cada GSE.

9 El rango etario de las madres fluctúa entre los 30 y 39 años. 


\section{TABLA 1}

Matriz de análisis

\begin{tabular}{|c|c|c|c|c|c|c|c|c|}
\hline $\begin{array}{l}\text { GRADA- } \\
\text { CIÓN }\end{array}$ & EJEMPLO & TIPO DE & \multicolumn{4}{|c|}{ ANÁLISIS DE TRANSITIVIDAD } & $\begin{array}{l}\text { RANGO } \\
\text { DEL } \\
\text { GRUPO }\end{array}$ & $\begin{array}{l}\text { RANGO } \\
\text { DE LA } \\
\text { PALABRA }\end{array}$ \\
\hline \multirow{2}{*}{$\begin{array}{l}\text { Cuantifica- } \\
\text { ción de } \\
\text { proceso: } \\
\text { Frecuencia }\end{array}$} & \multirow[t]{2}{*}{$\begin{array}{l}\text { yo lo veo } \\
\text { todos los } \\
\text { días }\end{array}$} & mental & yo & lo & veo & $\begin{array}{l}\text { todos los } \\
\text { días }\end{array}$ & \multirow[t]{2}{*}{$\begin{array}{l}\text { frase } \\
\text { nominal }\end{array}$} & \multirow{2}{*}{$\begin{array}{l}\text { adjetivo + } \\
\text { sustantivo } \\
\text { definido } \\
\text { plural }\end{array}$} \\
\hline & & & Sentidor & $\begin{array}{l}\text { Fenóme- } \\
\text { no }\end{array}$ & Proceso & $\begin{array}{l}\text { Circuns- } \\
\text { tancia }\end{array}$ & & \\
\hline
\end{tabular}

Las instancias de GRADACIÓN en las que participa el cuantificador todo fueron posteriormente contrastadas con los recursos del sistema de ACTITUD identificados en el co-texto, así como con los tipos de identidad materna descritos para el mismo corpus en Gajardo y Oteíza (2017), Gajardo (2018) y Gajardo (en prensa).

\section{Análisis y discusión}

En esta sección se presentan y discuten los resultados en torno al cuantificador todo como recurso de GRADACión a partir del análisis realizado. Los resultados se presentan según los significados o funciones discursivas codificados por todo en sus realizaciones particulares, partiendo desde las formas más congruentes hacia las menos congruentes, esto es, desde aquellos casos donde la función de todo es cuantificadora, coincidente con la descripción gramatical tradicional del cuantificador, a las ocurrencias en las que el cuantificador cumple otra función discursiva.

\subsection{Cuantificación de Frecuencia}

En términos del sistema discursivo-semántico de GRADACIÓN, uno de los significados que codifica el cuantificador todo es de Fuerza alta por Cuantificación de Frecuencia. En los ejemplos que presentaremos a continuación lo que se cuantifica en términos de frecuencia es el conjunto completo de las entidades designadas por el sustantivo, en este caso, "los días":

(1) "Te empezái a cuestionar tu misma como "qué fue lo que paso, hice algo mal yo" o sea y eso en el día a día, no es tampoco algo que uno no lo VE, uno lo ve todos los días, yo veo todos los días qué estuvo bien, qué estuvo mal y hago como una reflexión, qué cosa puedo mejorar, que cosa no, o qué cosa está funcionando bien, si es que lo mantengo”. (F_GSEM ${ }^{10}$ )

(2) “Bueno además me pasaba la cuestión esta como que yo iba todos los días a la hora de almuerzo a sacarme leche también, ¿cachái? hasta los 6 meses (...) Igual me iba a almorzar, me

10 Al final de cada ejemplo se codifica el nombre de la participante, en este caso, únicamente la inicial, seguido por el grupo socioeconómico (GSE) al que pertenece, esto es, alto (A), medio (M) o bajo (B). 
iba a almorzar a la casa, igual como que tenía la costumbre, ¿cachái? y pa mí era mucho más

importante mi hijo. Sí, yo me iba todos los días, me iba a almorzar siempre a mi casa a ver a Andrés". (M_GSEA)

En los dos ejemplos precedentes, todo se realiza en el rango de la cláusula como una Circunstancia temporal y en el rango del grupo como una frase nominal, constituida por [todos (adjetivo) + sustantivo definido plural]. Desde una perspectiva discursivo-semántica, "todos” (“todos los días”) tiene la función de cuantificar los procesos en términos de cuán frecuentemente ocurren. En estos ejemplos, se señala una frecuencia alta. Estas instancias indican que todo funciona para cuantificar la totalidad de los miembros del conjunto denotado por el grupo nominal, en este caso, se refiere al total de los "días". En otras palabras, el cuantificador tiene el significado de hacer referencia al conjunto integral de los componentes que constituyen su denotación (NGLE 2009).

En términos valorativos, el cuantificador todo, como recurso de Cuantificación de frecuencia, colabora con la evocación de valoraciones positivas relacionadas con la identidad materna. En el primer ejemplo, la madre cuestiona su rol materno "todos los días", evocando así un Juicio positivo respecto de su capacidad para mejorar su práctica materna desde la reflexión ${ }^{11}$. El segundo ejemplo, por otra parte, muestra los esfuerzos de una madre por almorzar "todos los días" en su casa para poder estar con su hijo, GRADACión que también evoca un Juicio positivo en relación a la tenacidad de la madre ${ }^{12}$.

\subsection{Cuantificación por Alcance: Tiempo}

Otro significado de GRADACión que realiza el cuantificador todo se relaciona con la Cuantificación por Alcance en términos de tiempo. En los ejemplos (3) y (4), aquello que se cuantifica es la extensión del proceso en cuanto a su alcance en tiempo ("todo el rato estoy preocupada", "quería estar todo el día en mis brazos"):

(3) "Estoy vinculada las 24 horas del día, me entiendes que es distinta a las otras relaciones que uno tiene, que tú estás trabajando y no estás pendiente de cómo esta tú marido/ no, todo el rato estoy preocupada de Pablo [su hijo], todo el rato, o sea llamo en la mańana y en la tarde, ponte para hacerte una idea entonces". (B_GSEA)

11 En Gajardo (2018) se muestra la manera en que la acumulación de Juicios de Capacidad e instancias de GRADACIón de Cuantificación (como es el caso de "todos los días") forman patrones valorativos que son claves para la construcción de una identidad de madre reflexiva en el grupo socioeconómico medio. Este tipo de subjetividad es común en madres de clase media, quienes han vivido un importante proceso de secularización y pluralización (Yopo Díaz 2016), por lo que tienden a cuestionar modelos maternos tradicionales y sus propias prácticas maternas.

12 En Gajardo (en prensa) se discuten los patrones valorativos que conforman una identidad de madre presente, donde la GRADACIÓN de Cuantificación también tiene un papel importante. En este sentido, el cuantificador todo en el ejemplo contribuye a una autorepresentación de madre que es capaz de realizar sacrificios para estar "todos los días" con su hijo. 
(4) "Quería [su hija] estar conmigo porque era muy apegada (...) cuando chica era regalona, quería estar todo el día en mis brazos”. (A_GSEB)

En estos ejemplos, la función de todo es distinta a las presentadas anteriormente, ya que lo que se cuantifica no es la frecuencia, sino la extensión del proceso, es decir, se amplifica un fenómeno en términos de su prolongación en el tiempo. En ambos ejemplos, el cuantificador todo se realiza a nivel clausular como una Circunstancia de tiempo y en el rango del grupo como un grupo nominal, compuesto por [todo (adjetivo) + sustantivo definido singular]. Se diferencia de "todos los días", caso presentado anteriormente, ya que en el plural significa "todos y cada uno de los días en el período que se toma en consideración", mientras que, en el singular, todo cuantifica "como un bloque el objeto designado" (Alarcos 2000: 127), de modo que "todo el día" es sinónimo de "el día entero".

El cuantificador todo como recurso de GRADACIÓN en estos casos también está involucrado en la evocación de valoraciones positivas que colaboran con la construcción de la identidad materna. En el ejemplo (3), "todo el rato" en conjunto con otros elementos valorativos, tales como "estoy vinculada las 24 horas" y "estoy preocupada", evoca un Juicio positivo de Integridad Moral, contribuyendo a la formación de una identidad de madre presente $^{13}$ en las madres del GSE alto. Asimismo, en el ejemplo (4) el recurso de cuantificación "todo el rato", colabora con la construcción de una identidad de madre apegada ${ }^{14}$, característica del GSE bajo.

\subsection{Cuantificación de Entidades cuya referencia es anafórica}

En los siguientes ejemplos, el cuantificador todo funciona para graduar entidades—no así procesos- cuyas identidades se recuperan en el co-texto. En el corpus, se presentaron dos ocurrencias distintivas de este recurso de Cuantificación. En primer lugar, se observaron casos donde todo tiene un uso pronominal ("todo fluía") como muestra el ejemplo (5) y, en segundo término, surgieron instancias en las que todo tiene un uso adjetivo y va acompañado del pronombre neutro eso ("todo eso"), como sucede en el ejemplo (6):

(5) "Estaba como loca así, me deprimí y sí, empecé con terapia también ahí, porque fue más que, quizás era una depresión, pero la sensación era como de desbordada de emociones, y cuando me iba a clases me iba con la cabeza enredadísima, me costaba pensar todo, juraba ${ }^{15}$ que no

13 Por restricciones de espacio, solo presentamos un ejemplo para observar la manera en que opera el cuantificador todo en la evocación de valoraciones. Recordemos que las estrategias valorativas funcionan por acumulación, por lo que con solo un ejemplo no se pueden apreciar los patrones que dan forma a construcciones identitarias particulares. Para mayor información en cuanto a los tipos de identidad materna encontradas en el grupo socioeconómico alto, cf. Gajardo (en prensa).

14 En Gajardo y Oteíza (2017), se discute cómo las madres del grupo socioeconómico bajo construyen una identidad de madre apegada, a través de las acciones que realizan sus hijos/as para estar con ellas, donde el cuantificador todo tiene un rol clave en la expresión del grado máximo de apego entre madre e hijo/a.

15 En español de Chile, "jurar" se utiliza coloquialmente para significar "creer firmemente". 
iba a poder hacer la clase, a ese nivel, y llegaba y todo fluía como antes súper bien y la clase me

ayudaba a como calmar todo, era súper buena y aparte se me iban quitando las aprensiones". (F_GSEM)

En este ejemplo, dos de las tres ocurrencias de todo se presentan en cláusulas materiales, donde el cuantificador se realiza como un participante Meta ("calmar todo", "todo fluía"), mientras que en la cláusula mental "me costaba pensar todo", se realiza como un participante Fenómeno. En el rango de las palabras, todo funciona como un pronombre indefinido. En términos discursivo-semánticos, lo que se cuantifica en el primer y segundo caso ("pensar todo", "todo fluía") es lo relativo a la "cla$s e$ "16, referente que se obtiene del co-texto. En el último caso ("calmar todo"), todo hace referencia a estar "desbordada de emociones" y a estar "con la cabeza enredadísima", que corresponden a los sentimientos negativos que experimentaba la participante y que se "calmaban" luego de dictar su clase. En estos ejemplos, todo tiene un significado genérico, que puede ser sinónimo de "todas las cosas" y designa a la totalidad de los miembros del conjunto referidos en el co-texto (NGLE 2009).

$\mathrm{Al}$ igual que en las ocurrencias descritas anteriormente, todo también tiene una función valorativa, en tanto colabora con la evocación y cuantificación de algunas actitudes. En el relato se muestra la manera en que los roles de madre y de mujer/trabajadora están en constante tensión ${ }^{17}$ en una madre del GSE medio, lo que se expresa mediante diversos Juicios negativos referidos a la capacidad de compatibilizar las actividades laborales con las maternas ("me costaba pensar en todo", "juraba que no iba a poder hacer la clase"). Cuando la madre comienza a hacer sus clases, se volvía a sentir "como antes" (antes de convertirse en madre) y la angustia desaparecía. En ambos casos, todo colabora con la cuantificación y evocación de actitudes negativas y también con aquellas positivas generadas luego de reencontrarse con su rol de trabajadora, que se expresan mediante Apreciaciones positivas ("y llegaba y todo fluía") y Afectos de Seguridad ("la clase me ayudaba como a calmar todo").

Otro ejemplo en el que el cuantificador todo cuantifica entidades es el que introducimos a continuación:

(6) Yo pago todo, luz, el gas, mercadería todo, colaciones de mi hijo, su plata, todo eso yo, así que prácticamente pesqué yo las riendas, soy yo la que decide". (E_GSEB)

El cuantificador todo se realiza en una cláusula material como un participante Meta y como un grupo nominal compuesto por [todo (adjetivo) + pronombre demostrativo] en el rango de las palabras. En este ejemplo particular, lo cuantificado es "eso", que hace referencia a la totalidad de los servicios y alimentos que la madre paga por su hijo, tales como "la luz", "el gas", "las colaciones", elementos que se recuperan anafóricamente en el co-texto. Al igual que en el ejemplo (5), el cuan-

16 La madre dicta clases de arte.

17 En Gajardo (2018) se discute la manera en que las madres del GSE medio, debido a su capacidad reflexiva, establecen una línea divisoria entre la identidad materna y la femenina, fomentado una construcción identitaria de madre y mujer en constante tensión. En este sentido, el cuantificador todo colabora con la evocación de valoraciones involucradas en esta identidad materna. 
tificador opera sobre el total de los elementos mencionados previamente en el discurso. También

en este ejemplo, todo como recurso de GRADACIÓn tiene una función valorativa, ya que aumenta la fuerza de las actitudes y permite, en conjunto con otros elementos, tales como la metáfora "pesqué yo las riendas", evocar un Juicio positivo respecto de la capacidad de la madre para hacerse cargo de las necesidades de su hijo ${ }^{18}$.

\subsection{Cuantificación de Entidades cuya referencia es homofórica}

El cuantificador todo aparece también realizando GRADACIÓn de Fuerza como Cuantificación de entidades cuya identidad ya no se recupera en el co-texto, como en los ejemplos (5) y (6) arriba, sino en el contexto de cultura, esto es, tomando como base el conocimiento cultural que comparten los interlocutores. A esto se le ha llamado referencia homofórica (Martin y Rose 2007). Cumpliendo esta función discursiva, en nuestro corpus encontramos todo funcionando como pronombre indefinido, acompañado o no de preposición, como muestran el ejemplo (7) a continuación:

(7) “No, es que como es la primera [guagua ${ }^{19}$ ] estoy como improvisando/ más como aprendiendo/ como más improvisada/ trato de ser como más sistemática pero no, a veces tengo todo muy organizado pero a veces muy a lo que salga, por ahora como espontáneo”. (F_GSEM)

En este ejemplo, todo participa como Posesión al interior de una cláusula relacional posesiva, y como un pronombre indefinido en el rango de la palabra, acompañado de una Circunstancia de manera ("muy organizado"). A nivel discursivo-semántico, el cuantificador realiza una Cuantificación de entidades que se recuperan del contexto de cultura, a partir del cual se puede inferir que todo se refiere al "conjunto de aspectos que concierne al cuidado de los hijos". En conjunto con este recurso de Cuantificación, podemos apreciar también una Intensificación de cualidad en la Circunstancia de manera ("muy organizado"), seguida de un significado de contra-Expectativa ("pero") y una prosodia que irradia valores de Foco ("como aprendiendo", "como más improvisada", “como espontáneo"), elementos que contribuyen a la conformación de una identidad materna cuya habilidad de integrar los aprendizajes y experiencias a su quehacer para flexibilizar la maternidad, da cuenta de una madre en proceso de construcción ${ }^{20}$, característica del GSE medio.

El ejemplo (8) a continuación muestra también una cuantificación de entidades cuya referencia se recupera con base en el conocimiento cultural compartido por las interlocutoras:

18 En Gajardo y Oteíza (2017) se discute cómo las madres del grupo socioeconómico bajo realizan acciones sacrificadas destinadas a la provisión de recursos fundamentales para sus hijos/as, de modo de que no experimenten ningún tipo de necesidad. Esto se expresa por medio de estrategias valorativas particulares, donde la cuantificación de dichas acciones codificadas en el cuantificador todo tienen un rol fundamental y contribuyen a la construcción de una identidad de madre luchadora.

19 "Guagua" en español de Chile significa "bebé".

20 En Gajardo (2018) se discute cómo las madres del grupo socioeconómico medio construyen una identidad incompleta, es decir, una madre en proceso de construcción. 
(8) "entonces hacís como de todo, y las niñitas te llaman y "puedo ir a la casa de la amiguita",

y "podís ir al supermercado" y "podís hacer esto" y además trabajái, como que hacís todo al final”. (J_GSEA)

En este ejemplo, de todo opera como participante Meta de una cláusula material, instanciado como un grupo nominal compuesto por [de (preposición) + todo (pronombre indefinido)] en el rango de la palabra. Este tipo de construcciones se describen tradicionalmente como expresiones pseudo-partitivas que denotan "suficiencia", y que hacen referencia a un conjunto de cosas que se interpreta como consabido (NGLE 2009: 2.321). Como en el ejemplo (7), este conjunto de entidades cuantificadas remite a los aspectos relacionados con el ejercicio de la maternidad, lo que se relaciona también con la presentación de una identidad de madre integral. Esta identidad se construye a través de estrategias valorativas que incluyen la enumeración de las acciones sacrificadas que las madres realizan para estar presentes para sus hijos y la presentación de una madre con una gran capacidad para llevar a cabo múltiples actividades y cumplir satisfactoriamente en todas ellas ${ }^{21}$.

Esta representación identitaria se refuerza prosódicamente mediante la presencia de otro proceso material ("como que hacís todo al final”), donde todo está cuantificando entidades recuperables en el co-texto ("hacer esto", "además trabajái”).

En nuestros datos también encontramos una forma particular de Cuantificación de entidades de referencia homofórica, como parte de complejos clausales, tal como puede verse en el ejemplo (9), a continuación:

(9) “[La maternidad] en una manera ha significado menos autonomía y menos libertad (...) esto de la responsabilidad, de tener a alguien a quien cuidar, es increíble, y aparte, bueno, todo lo que es tener un hijo que es como indescriptible tener un hijo, pero un hijo es una cosa muy especial la verdad muy es como una alegría y un amor un amor un amor que nunca había sentido antes". (B_GSEA)

En este ejemplo, "todo lo que", así como la cláusula incrustada "tener un hijo" operan como participantes en un proceso relacional ("es"). En el rango del grupo el cuantificador todo forma parte de un grupo nominal compuesto por [todo (pronombre indefinido) + lo (pronombre átono) + que (relativo)] en el rango de la palabra. El proceso relacional de identificación indica una relación simbólica entre dos elementos de diferentes órdenes de abstracción (Martin, Matthiessen y Painter 2010), es decir, un Valor (“todo lo que”) con una Ocurrencia (o Token) ("tener un hijo"). La Cuantificación de entidades opera sobre los ámbitos involucrados en esa relación simbólica,

21 Confrontar Gajardo (en prensa), donde se discute esta identidad de madre integral, propia del grupo socioeconómico alto de Santiago de Chile, en la cual se entremezclan significados de sacrificio, extenuación y, al mismo tiempo, una gran capacidad para lograr todos sus cometidos. En esta construcción identitaria, "de todo" y "todo" tienen un rol fundamental, por cuanto maximizan las acciones sacrificadas realizadas por las madres del GSE alto en pos de estar presentes para sus hijos/as. 
es decir, sobre "todo aquello que involucra o implica tener un hijo". Nuevamente, como en los ejemplos (7) y (8), la referencia de esas entidades debe recuperarse recurriendo al conocimiento cultural de los participantes.

En términos valorativos, las actitudes relacionadas con estos elementos cuantificados ("indescriptible", "una cosa muy especial", "una alegría", "un amor") expresan apreciaciones y afectos que se relacionan con la construcción discursiva de los significados positivos de la maternidad y con una identidad de madre integral ${ }^{22}$, por medio de la cual las madres posicionan a sus hijos como prioridad y como una fuente de amor y felicidad, pese a que les haya significado una pérdida en términos de autonomía.

\subsection{Fuerza de Intensificación}

Un significado menos congruente que codifica el cuantificador todo corresponde al de Fuerza como Intensificación. En este sentido y, a diferencia de los ejemplos precedentes, todo como recurso de Intensificación no cuantifica entidades o procesos, sino que amplifica el grado de las valoraciones expresadas, como ocurre en los ejemplos que se incluyen a continuación:

(10) "En ese sentido somos juguetones y él es bien cariñoso conmigo, es que ya como está grande se da cuenta, pero él siempre ha sido así conmigo, bien cariñoso, y bueno, con la que es más apegada es conmigo po, siempre, siempre ha sido apegado conmigo/ es que él es todo". (E_GSEB)

(11) "Yo a mi hija la adoro, pa mí es todo/ ella es mi regalona/ después ya el señor me mandó una bendición que es el Cristopher, que ese también es mi conchito ${ }^{23} /$ bueno pa mí, mis hijos son todo/ son todo". (O_GSEB)

En estos dos ejemplos, todo ocurre en una cláusula relacional atributiva, donde los hijos/as son el Portador y el cuantificador se realiza como un Atributo, funcionando como un pronombre en el rango del grupo. A nivel discursivo-semántico, todo se encuentra lexicalizado como un atributo ("pa mi es todo", "mis hijos son todo", "él es todo") y constituye un recurso de Intensificación de Cualidad, mediante infusión, que al mismo tiempo realiza la inscripción de un Afecto de Felicidad. Es decir, se trata de la fusión de un significado de GRADACión con uno valorativo en un mismo término (Martin y White 2005). Asimismo, en estas cláusulas atributivas, todo realiza lo que Martin y White (2005: 142) denominan "maximizador", puesto que consiste en una "locución que construye la máxima intensidad posible”, al encontrarse en el extremo de la escala de intensificación. En ambos relatos, todo como recurso de Intensificación, colabora con la creación de una prosodia afectiva, en conjunto con diversas inscripciones de Afectos de Felicidad, realizados a través de los atributos "cariñoso", "apegado", "conchito", entre otros, y diversos recursos de GRADACIÓn de Fuerza

22 Al igual que en el ejemplo (8), se puede confrontar Gajardo (en prensa) para una discusión de los elementos que conforman una identidad de madre integral en el grupo socioeconómico alto de Santiago de Chile.

23 "Conchito" se refiere al hijo menor en español de Chile. 
alta ("más", "siempre", "bien"). Por lo tanto, este significado de todo tiene una función intensifica-

dora, que permite amplificar el grado de las posiciones expresadas, que en este caso dicen relación con una carga afectiva, contribuyendo a la creación de una identidad de madre apegada ${ }^{24}$.

\subsection{Foco de Especificidad: Autenticidad}

El cuantificador todo también realiza significados menos congruentes que aquellos de cuantificación e intensificación descritos anteriormente, es decir, se utiliza igualmente para graduar en términos de Foco. En los ejemplos siguientes describiremos este recurso usado para graduar una categoría semántica en cuanto a sus niveles de prototipicidad, esto es, según cuánto concuerda con los rasgos típicamente asociados a una categoría particular:

(12) "Capté que en el fondo, a propósito de los cambios que uno tiene que hacer como con esta nueva identidad, caché que ya no podía trabajar tanto, yo estaba trabajando mucho y eso fue todo un proceso porque fue instalarme en otro lado, fue aumentar la consulta porque yo tenía un poquito (...)”. (B_GSEA)

Este ejemplo muestra la realización del cuantificador en una cláusula relacional atributiva, en la que "todo un proceso" se realiza como un Atributo y, en el rango de grupo, como un grupo nominal constituido por [todo (adjetivo) + un (artículo indefinido) + sustantivo abstracto]. Como recurso de GRADACIÓn de Foco, todo agudiza o especifica que la situación vivida ${ }^{25}$ cumple con los atributos para ser considerada auténticamente como "un proceso". Es significativo señalar que "un proceso" no tiene un significado valorativo intrínseco, pero al ser graduado (agudizado) por todo, se vuelve valorativo y, de este modo, evoca una Apreciación negativa de Valoración Social. Como señalan Martin y White (2005: 139), cuando un término que no es valorativo se gradúa en términos de Foco, "existe una fuerte tendencia a que la escala de prototipicidad adquiera actitudinalidad". Si bien en este relato se evoca una valoración negativa, en el ejemplo (13), la GrADACión en Foco permite evocar una actitud positiva:

(13) “Si no estarían las niñitas, qué haría, qué lata, qué qué, le da como toda una trascendencia, todo un sentido de vida”. (J_GSEA)

En este ejemplo, se observan dos ocurrencias consecutivas de este recurso de GRADACIÓn de Foco según prototipicidad. En este caso particular, lo graduado es la maternidad ("si no estarian las niñi-

24 Es importante señalar que esta construcción atributiva donde todo tiene una función intensificadora, solo ocurre en el grupo socioeconómico bajo. Resulta un elemento valorativo clave en la formación de una identidad de madre apegada en el discurso de participantes pertenecientes este estrato.

25 En este caso, la situación vivida corresponde a la disminución de la carga laboral de una madre del grupo socioeconómico alto, debido a la dificultad de compatibilizar la práctica profesional con la materna. 
tas"), que cumple con los rasgos para ser considerada como una práctica que deja verdaderamente

"una trascendencia" y "un sentido de vida". A diferencia del ejemplo anterior ("todo un proceso"), en este relato todo está involucrado en la evocación de valoraciones positivas. Ahora bien, "sentido de vida", a diferencia de "trascendencia" ya es un término valorativo que inscribe una Apreciación positiva de Valoración Social. Todo contribuye a reconstruir esta valoración, en tanto la evalúa como un miembro auténtico de la categoría sentido de vida. Por lo tanto, este recurso de GRADACión de Foco actúa tanto sobre significados valorativos como no valorativos.

Como pudimos observar en ambos ejemplos-(12) y (13) — esta construcción compuesta por todo acompañado de artículo indefinido y sustantivo abstracto, se utiliza para señalar que un objeto o persona "cumple con todos los atributos que se asocian a cierto prototipo". Como se trata de un uso valorativo, "estos sustantivos permiten ponderar una cosa o persona para hacerla objeto de consideración o enjuiciamiento" (NGLE 2009: 2.269). Por lo mismo, sería redundante añadirle a la construcción un término valorativo similar como los adjetivos verdadero o auténtico (NGLE 2009; Hood 2010). Es importante señalar que esta función se registró únicamente en los relatos de las madres del GSE alto, y está involucrada en la construcción de una madre autónoma y de una madre presente. ${ }^{26}$

\subsection{Foco de Completitud}

Finalmente, en nuestros datos todo aparece también realizando GRADACIón como Foco de completitud sobre significados actitudinales, tal como puede verse en el ejemplo (14) a continuación:

(14) "es que mi pareja no la ha criado/ YO la estoy criando (...) él justo no le tocaba, faltó para que yo pudiera trabajar y cuando llegué, no poh, estaba todo emputecido y enojado y era el primer día que la cuidaba”. (A_GSEB)

En términos de transitividad, todo participa como Atributo en una cláusula relacional atributiva, y forma parte de un grupo adjetivo constituido por [todo (adverbio) + adjetivo] en el rango de la palabra. Se ha descrito que, en conjunto con adjetivos o participios, todo funciona como adverbio de grado con el significado de "enteramente o por completo" (NGLE 2009). A nivel discursivo-semántico, todo funciona graduando un significado actitudinal intensificado mediante infusión ${ }^{27}$. Este tipo de premodificación de significados actitudinales ha sido descrito por Hood (2010) como Foco de completitud, en el sentido de reforzar o suavizar los límites de un significado categorial. En este caso, la GRADACión de Foco como completitud refuerza los límites categoriales del atributo, indicando que el significado del mismo se realiza en su totalidad.

La valoración negativa que se construye en este ejemplo sobre un actor distinto de la madre (en este caso, el padre), contribuye a una presentación identitaria materna con base en el sacrificio y en

26 Confrontar Gajardo (en prensa) en relación a los tipos de identidad materna encontradas en el GSE alto de Santiago de Chile.

27 "Emputecido/a" se utiliza en español de Chile de manera coloquial para expresar "muy enojado/a". 
la entrega total, que le atribuye a la madre toda la responsabilidad relativa al cuidado y bienestar ma-

terial y psicológico de su hijo. Esta estrategia valorativa le permite a la madre construirse como una madre luchadora, construcción característica del grupo socioeconómico bajo de Santiago de Chile ${ }^{28}$. El contenido actitudinal reverbera no solo en la gradación de un significado actitudinal ya intensificado, sino también mediante intensificación por repetición: "estaba todo emputecido y enojado".

Como se observó a lo largo de esta sección, el cuantificador todo resultó ser un recurso de GRADACIÓN muy productivo, que realiza significados congruentes con su descripción gramatical tradicional, esto es, cumple una función cuantificadora. Asimismo, todo despliega realizaciones menos congruentes, en las que cumple una función intensificadora de cualidades, como también la de graduar una categoría semántica en términos de prototicipidad y de completitud. En la figura 2 ofrecemos una red sistémica del cuantificador todo con respecto a sus significados de GRADACIÓN:

\section{FIGURA 2}

Red sistémica del cuantificador todo como recurso de GRADACIóN

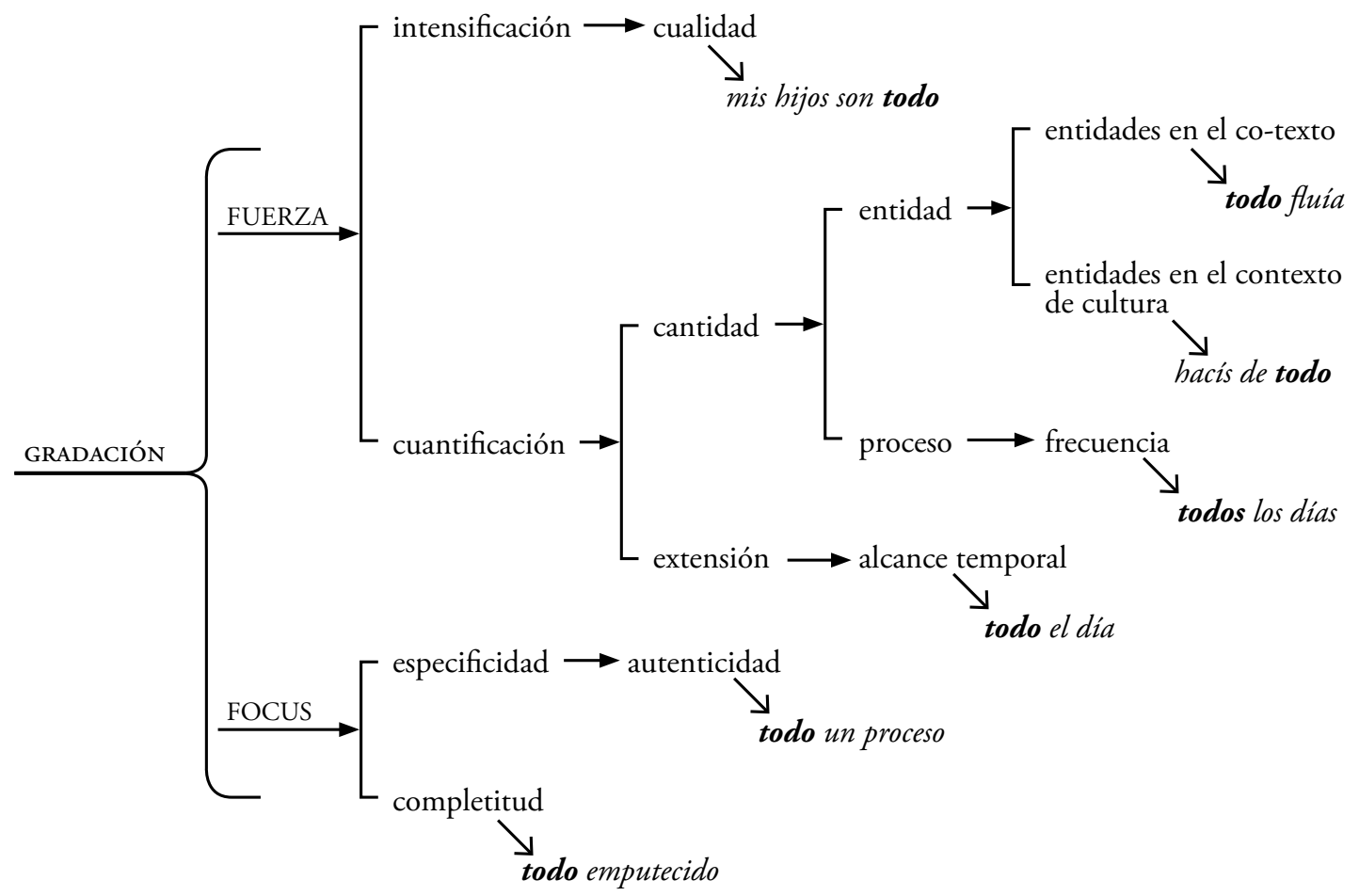

\section{Conclusión}

Este artículo analizó el funcionamiento del cuantificador todo como recurso que instancia gradaciones de valoraciones en un corpus de español oral compuesto por un grupo de madres de tres

28 Para una discusión en torno a las estrategias valorativas que conforman la identidad de una madre luchadora, confrontar Gajardo y Oteíza (2017). 
grupos socioeconómicos de Santiago de Chile. En primer lugar, este trabajo demuestra que las diversas realizaciones léxico-gramaticales de todo tienen un rol importante en la cuantificación, intensificación y agudización de los significados actitudinales en el campo de la identidad materna. Así, en términos de la realización de significados congruentes con su descripción gramatical (como cuantificador), todo funciona como Cuantificador de procesos y de entidades. En el caso de la Cuantificación de procesos, todo participa en la Cuantificación en términos de frecuencia ("todos los días") y en términos de la extensión temporal de procesos ("todo el día").

En relación a la Cuantificación de entidades, se describió el uso del cuantificador todo cuyos referentes se recuperan tanto en el co-texto a través de la referencia anafórica ("calmar todo", "todo eso") como en el contexto cultural más amplio, es decir, por medio de la referencia homofórica ("haces de todo", "todo lo que es tener un hijo").

Dentro de los significados menos congruentes que realiza todo, se describió su uso como recurso de Fuerza de Intensificación ("mis hijos son todo"). Finalmente se registraron dos realizaciones del cuantificador todo como recurso de Foco: graduando una categoría semántica en cuanto a sus niveles de prototipicidad ("todo un proceso"), y graduando significados en relación con su completitud ("todo emputecido").

Estas realizaciones de todo como recursos de GRADACIón tienen un rol significativo en la evocación de valoraciones, tanto positivas como negativas, involucradas en la construcción de identidades maternas particulares según el grupo socioeconómico de pertenencia. Mediante la presentación de acciones y atributos más o menos cuantificados, más o menos intensificados y más o menos agudizados, las participantes construyen una determinada representación de sí mismas y del ejercicio de su maternidad en relación con ciertos valores considerados estimables en sus comunidades de práctica.

La descripción tipológica de los recursos de GRADACIÓN desde una perspectiva trinocular nos permitió identificar cómo los significados discursivo-semánticos involucrados en la Cuantificación, Intensificación y Focalización de significados actitudinales e ideacionales se realizan mediante diversas selecciones a nivel léxico-gramatical, que manifiestan un despliegue de posibilidades en el uso del cuantificador todo en el rango de la cláusula, el grupo y la palabra. Tomando en consideración el co-texto, pudimos apreciar las potencialidades discursivo-semánticas de este recurso para participar en la construcción de significados interpersonales involucrados en la legitimación de posicionamientos identitarios con respecto a la maternidad de las participantes.

El propósito de este trabajo fue presentar una descripción comprehensiva de recursos léxico-gramaticales específicos para realizar GRADACIÓN en el campo de la identidad materna, lo que se enmarca en el interés mayor que, como analistas del discurso, tenemos en relación con la comprensión del potencial de recursos valorativos para construir identidades y posicionamientos discursivos en diversos campos de la actividad social.

\section{Referencias bibliográficas}

Achugar, M. 2008. What we remember: The Construction of Memory in Military Discourse. Amsterdam: John Benjamins Publishing Co.

Achugar, M. 2016. Discursive Processes of Intergenerational Transmission of Recent History. (Re) making our Past. New York: Palgrave MacMillan. 
AIM. 2015. Asociación Investigadores de Mercado. Nuevo modelo de grupos socioeconómicos: AIM.

Alarcos, E. 2000. Gramática de la Lengua Española. Real Academia Española. Colección Nebrija y Bello. Madrid: Espasa Calpe.

Alba Juez, L. \& Thompson, G. 2014. The many faces of evaluation. En G. Thompson \& Alba Juez, L. Evaluation in Context. Amsterdam y Philadelphia: John Benjamins.

Alcina Franch, J. \& Blecua, J.M. 1979. Gramática Española. Barcelona: Ariel.

Arce Castillo, A. 1999. Intensificadores en español coloquial. Anuario de Estudios Filológicos 22: 37-48.

Benwell, B. \& Stokoe, E. 2006. Discourse and Identity. Edinburgh: Edinburgh University Press.

Bertaux, D. 1989. Los Relatos de Vida en el Análisis Social. Historia y Fuente Oral 1: 87-96.

Bertaux D. 2005. Los Relatos de Vida: perspectiva etnosociológica. Barcelona: Bellaterra.

Bolívar, A. 1994. Discurso e interacción en el texto escrito. Consejo de Desarrollo Científico y Humanístico. Caracas: Universidad Central de Venezuela.

Bolívar, A. 2007. El análisis interaccional del discurso: el texto a la dinámica social. En A. Bolívar (Ed.). Análisis del Discurso ¿Por qué y para qué?, pp. 248-277. Caracas: Universidad Central de Venezuela.

Caffarel, A., Martin, J.R y Matthiessen C.M.I.M. 2004. Language Typology. A Functional Perspective. Amsterdam: John Benjamins Publishing Company.

Chouliaraki, L. \& Fairclough, N. 1999. Discourse in Late Modernity: Rethinking Critical Discourse Analysis. Edinburgh: Edinburgh U.P.

Ferraroti, F. 1988. Sobre a autonomia do método biográfico. En A. Nóvoa y M. Finger. (Eds.). O método (auto)biográfico e a formação. Lisboa: Ministério da Saúde, pp. 17-34.

Ferraroti, F. 2007. Las historias de vida como método. Convergencia 44: 15-40.

GajARdo, C. 2017. La construcción discursiva de la identidad de madre/trabajadora en un grupo de madres de tres niveles socioeconómicos de Santiago de Chile. (Tesis Doctoral). Pontificia Universidad Católica de Chile, Santiago, Chile.

GAJARDO, C. 2018. La construcción discursiva de la identidad de madre/ trabajadora en madres del grupo socioeconómico medio de Santiago de Chile. RALED 1: 118-137.

Gajardo, C. En prensa. Madres que hacen de todo. La construcción discursiva de madre trabajadora en el grupo socioeconómico alto de Santiago de Chile. Discurso \& Sociedad 15, 2.

Gajardo, C. \& Oteíza, T. 2017. The ideological construction of mother identity in the discourse of four women of the lower socio-economic group from Santiago, Chile. Discourse \& Society 28: 142-161. 
Giddens, A. 1991. Modernity and Self-Identity: Self and Society in the Late Modern age. Stanford:

Stanford University Press.

Giudice, J. 2010: Interpretación y re-construcción del período 1976-1983 en manuales de ciencias sociales argentinos. En O. Albahaca, M. KahWan y S. Serrón (Coords.). Memorias del V Congreso Internacional de la Cátedra UNESCO, Instituto Venezolano de Investigaciones Lingüísticas y Literarias. Andrés Bello - Cátedra UNESCO.

Gómez, V., Arellano, O. y Valenzuela, C. 2017. Negociaciones en familia: género, trabajo y cuidado en Chile. Revista Estudios Feministas 25, 2: 661-682.

Gonzálvez, H. 2016. El 'trabajo de parentesco' que realizan las familias en Santiago de Chile. Revista de Antropología Social 25, 1: 153-169.

Halliday, M.A.K. \& Matthiessen, C.M.I.M. 2014. Halliday's Introduction to Functional Grammar. London/New York: Routledge.

Hood, S. 2010. Appraising Research: Evaluation in Academic Writing. London: Palgrave Macmillan.

Hood, S. 2019. Appraisal. En G. Thompson, W. Bowcher, L. Fontaine \& D. Schönthal (Eds.). The Cambridge Handbook of Systemic Functional Linguistics, pp. 382-409. Cambridge: Cambridge University Press.

Hood, S. \& Martin, J.R. 2005. Invocación de actitudes: el juego de la gradación de la valoración en el discurso. Revista Signos 38, 58: 195-220.

Hunston, S. \& Thompson, G. 2000. Evaluation in Text. Oxford: Oxford University Press.

ICCOM. 2007. Descripción Básica de los Niveles Sociales Hogares Urbanos Región Metropolitana 2007. Santiago: ICCOM Investigación de Mercado.

Kaplan, N. 2012. La construcción discursiva de personajes en las noticias televisivas sobre eventos conflictivos: un análisis en el marco de la teoría de la valoración. Empiricism and Analytical Tools For 21 Century Applied Linguistics. Selected Papers from the Xxix International Conference of the Spanish Association of Applied Linguistics, pp. 337-346.

Kumar, A. 2019. Language Typology. En G. Thompson, W. Bowcher, L. Fontaine \& D. Schönthal (Eds.). The Cambridge Handbook of Systemic Functional Linguistics, pp. 767-792. Cambridge: Cambridge University Press.

LaChance Adams, S. 2014. Mad Mothers, Bad Mothers \& What a "Good" Mother Would do: the ethics of ambivalence. New York: Columbia University Press.

Laney, E., Lewis Hall, E., Anderson, T.L. \& Willingham, M.M. 2015. Becoming a Mother: The Influence of Motherhood on Women's Identity Development, Identity, 15, 2: 126-145.

Martin, J.R. 1992. English text: system and structure. Amsterdam: Benjamins.

Martin, J.R. 2000. Beyond Exchange: Appraisal Systems in English. En S. Hunston \& G. Thompson. (Eds.). Evaluation in Text. Oxford: Oxford University Press. 
Martin, J.R. 2014. Evolving systemic functional linguistics: beyond the clause. Functional Linguistics 1, 3: 1-24.

Martin, J.R. 2019. Discourse Semantics. En G. Thompson, G., W. Bowcher, L. Fontaine \& D. Schönthal (Eds.). The Cambridge Handbook of Systemic Functional Linguistics, pp. 358-381. Cambridge: Cambridge University Press.

Martin, J.R. \& White, P.R.R. 2005. The Language of Evaluation: Appraisal in English. New York: Palgrave Macmillan.

Martin, J.R. \& Rose, D. 2007. Working with Discourse: Meaning beyond the clause. London, New York: Continuum.

Martin, J.R., Matthiessen, C. \& Painter, C. 2010. Deploying Functional Grammar. Beijing: The Commercial Press.

Moliner, M. 2013. Gramática Básica del Español. Madrid: Gredos.

Muñoz, L.A., Sánchez, X., Arcos, E., Vollrath, A. y Bonatti, C. 2013. Vivenciando la maternidad en contextos de vulnerabilidad social: un enfoque comprensivo de la fenomenología social. Revista Latino-Americana Enfermagem 21,4: 1-7.

NGLE. 2009. Nueva Gramática de la Lengua Española. Real Academia Española. Asociación de Academias de la Lengua Española. Volumen I. Madrid: Espasa Libros.

Oтеíza, T. 2009. Solidaridad ideológica en el discurso de la historia: Tensión entre orienta- ciones monoglósicas y heteroglósicas. Revista Signos 42, 70: 219-244.

Отеíza, T. 2011. Representación de las memorias del pasado: intersubjetividad en el discurso pedagógico de la historia. En T. Oteíza y D. Pinto (Eds.). En (re)construcción: Discurso, identidady nación en los manuales escolares de historia y de ciencias sociales, pp. 129-172. Santiago: Cuarto Propio.

Oteíza, T. y Pinuer, C. 2012 Prosodia valorativa: construcción de eventos y procesos en el discurso de la historia. Discurso \& Sociedad 6, 2: 418- 446.

Oteíza, T. y Pinuer, C. 2013. Valorative prosody and the symbolic construction of time in recent national historical discourses. Discourse Studies 15, 1: 43-64.

Oteíza, T. \& Pinuer, C. 2016. Appraisal framework and critical discourse studies: a joint approach to the study of historical memories from an intermodal perspective. International Journal of Language Studies 10, 2: 5-32.

Oteíza, T. y Pinuer, C. 2019. El sistema de valoración como herramienta teórico-metodológica para el estudio social e ideológico del discurso. Logos 29, 2: 207- 229

Pascual, M. 2014. La asociación de recursos de valoración: el caso de las organizaciones de derechos humanos en la prensa escrita argentina. Revista Onomázein 1, 29: 99-114.

Pascual, M. 2017. La violación a los derechos humanos en la Argentina: dinámica evaluativa de dos décadas de representación en medios. Discurso \& Sociedad 11, 3: 388-413. 
Rasse, A., Salcedo, R. \& Pardo, J. 2009. Transformaciones económicas y socioculturales:

¿Cómo segmentar a los chilenos hoy? En A. Joignant \& P. Güell. (Eds.). El arte de clasificar a los chilenos: En-foques sobre los modelos de estratificación en Chile, pp. 17-36. Santiago: Universidad Diego Portales.

SuÁrez Fernández, M. 2012. El comportamiento pragmático del cuantificador todo/s en castellano medieval. En T.E. Jiménez, B. López, V. Vásquez, A. Veiga y G. Rojo (Coords.). Cum corde et in nova grammatica: estudios ofrecidos a Guillermo Rojo, pp. 819-830. Santiago de Compostela: Universidade de Santiago de Compostela, Servizo de Publicacións e Intercambio Científico

Undurraga, R. 2013. Mujer y trabajo en Chile: ¿qué dicen las mujeres sobre su participación en el mercado laboral? En C. Mora (Ed.). Desigualdad en Chile: la continua relevancia del género, pp. 113-141. Santiago de Chile: Ediciones Universidad Alberto Hurtado.

VIan Jr., O. 2008. Appraisal System in Brazilian Portuguese: Resources for Graduation. Odense Working Papers in Language and Communication 29: 808-824.

Yopo DíAz, M. 2016. Enacting motherhood: time and social change in Chile. Journal of Gender Studies 27, 4: 411-427.

CONSUELO GAJARDO es Doctora en Lingüística de la Pontificia Universidad Católica de Chile y es profesora asistente en la misma universidad. Sus líneas de investigación son los Estudios del Discurso, Estudios Críticos del Discurso y el Sistema de la Valoración. Actualmente es co-investigadora del Proyecto Fondecyt Regular 1200003 "La atenuación lingüística en el español chileno: enfoque pragmalingüístico y sociopragmático”.

Correo electrónico: cgajardom@uc.cl

CLAUDIA CASTRO es antropóloga social y magister en Lingüística. Sus áreas de interés incluyen los estudios sociales y críticos del discurso y la escritura académica en la educación superior. Actualmente se desempeña como personal técnico en el Proyecto Fondecyt Regular 1170331, "El lenguaje de la valoración en español: descripción y sistematización de recursos para construir intersubjetividad”. Además, enseña el curso de Análisis de Discurso en la Universidad Alberto Hurtado, en Santiago de Chile.

Correo electrónico: claudiacastroa@gmail.com 